International Journal of Medical Sciences

ISSN 1449-1907 www.medsci.org 2008 5(6):341-346

Short Research Communication

(C) Ivyspring International Publisher. All rights reserved

\title{
XeNA: Capecitabine Plus Docetaxel, With or Without Trastuzumab, as Pre- operative Therapy for Early Breast Cancer
}

\author{
Stefan Glück ${ }^{1}$, Edward F. McKenna Jr², Melanie Royce ${ }^{3}$ \\ 1. Miller School of Medicine, University of Miami, Miami, FL, 33136, USA \\ 2. Medical Affairs Oncology, Roche, Nutley, NJ 07110-1199, USA \\ 3. University of New Mexico Cancer Research and Treatment Center, Albuquerque, NM, 87131, USA
}

$\triangle$ Correspondence to: Stefan Glück, MD, PhD, Professor of Medicine, Associate Division Chief Clinical Affairs, Division of Hematology/Oncology, Clinical Director, Braman Family Breast Cancer Institute, UMSylvester Comprehensive Cancer Center, University of Miami, Miller School of Medicine, 1475 NW 12th Ave., Suite 3510, Miami FL 33136. Tel: 305-243-4909; Fax: 305-243-4047; E-mail: SGluck@med.miami.edu.

Received: 2008.09.05; Accepted: 2008.11.03; Published: 2008.11.04

Combinations of capecitabine and a taxane are highly active in metastatic breast cancer, and synergy between capecitabine and docetaxel has also been demonstrated. Such combinations potentially would provide a promising non-anthracycline-based alternative for patients with early breast cancer. Non-anthracycline preoperative regimens are a particularly interesting proposition in human epidermal growth factor receptor 2 (HER2)-positive breast cancer, as they offer less cardiotoxicity and thus can be used concomitantly with preoperative trastuzumab therapy. Capecitabine plus docetaxel (XT) and trastuzumab with XT (HXT) are promising non-anthracycline regimens for the preoperative treatment of women with HER2-negative and HER2-positive breast cancer, respectively. The Xeloda in Neoadjuvant (XeNA) trial, an open-label, multicenter, phase II study, independently assesses the efficacy of preoperative XT in HER2-negative and HXT in HER2-positive breast cancer. A particularly important feature of the XeNA study is the use of pathologic complete response (pCR) plus near pCR (npCR) as the primary endpoint. pCR is associated with long-term survival, and although it is valuable as a surrogate marker, $\mathrm{pCR}$ has some limitations. Measurement of residual breast cancer burden (RCB) has been proposed as a more practical alternative to predict survival after preoperative chemotherapy. The combination of RCB-0 and RCB-I (npCR) expands the subset of patients shown to benefit from preoperative chemotherapy, and achievement of $\mathrm{pCR}$ or $\mathrm{npCR}$ is associated with long disease-free survival. In XeNA, the sum of pCR and npCR will facilitate correlative studies designed to identify patients most likely to benefit from XT and HXT and may expedite the clinical evaluation of these novel preoperative regimens.

Key words: Pathologic complete response, Breast-conserving surgery, Taxane, Anthracycline-induced cardiotoxicity

\section{INTRODUCTION}

Primary administration of systemic chemotherapy is standard of care for locally advanced breast cancer (LABC) [1]. In women with large operable tumors, preoperative administration might increase the likelihood of breast-conserving surgery [2]. Several randomized trials comparing preoperative versus postoperative treatment have shown equivalent disease-free survival (DFS) and overall survival (OS) [3]. Although pathologic stage may not correlate perfectly with DFS and OS, the value of findings from preoperative chemotherapy outweighs this disadvantage. Preoperative treatment serves as an excellent in vivo model, providing prognostic and potentially predictive information and facilitating the evaluation of tu- mor biomarkers to improve individualization of therapeutic strategies [4]. This may expedite the clinical development of new drugs and regimens.

\section{Pathologic response and clinical outcomes}

Pathologic complete response $(\mathrm{pCR})$ is associated with long-term survival [2,5-9]. However, despite its value as a surrogate marker, $\mathrm{pCR}$ has some limitations as an endpoint because of the variety in definitions between studies [10]. An international panel recommended that $\mathrm{pCR}$ must not include any residual invasive or in situ cancer in the breast or lymph nodes [1], but it has been argued that such a restrictive and purist definition weakens the clinical utility of this endpoint and diminishes the number of informative patients [11]. 
An interesting concept with important clinical implications is the attainment of significant tumor downsizing to microscopic levels only. Patients with small (T1a-b), node-negative breast tumors have excellent rates of long-term relapse-free survival $(91 \%$ at 10 years and $87 \%$ at 20 years) [12]. Measurement of residual breast cancer burden (RCB) has also been proposed as a practical alternative to the traditional dichotomy between $\mathrm{pCR}$ and residual disease to predict survival after preoperative chemotherapy (Figure 1) $[13,14]$. The combination of RCB-0 (pCR or American Joint Committee on Cancer stage 0 [15]) and RCB-I (near pCR [npCR]) expands the subset of patients shown to benefit from preoperative chemotherapy. Achievement of $\mathrm{pCR}$ or $\mathrm{npCR}$ is associated with long DFS, whereas moderate or extensive residual disease predicts for short DFS. Based on these data, reduction of the initial tumor size to T1a is used to define npCR in the Xeloda in NeoAdjuvant (XeNA) study of preoperative chemotherapy described below.

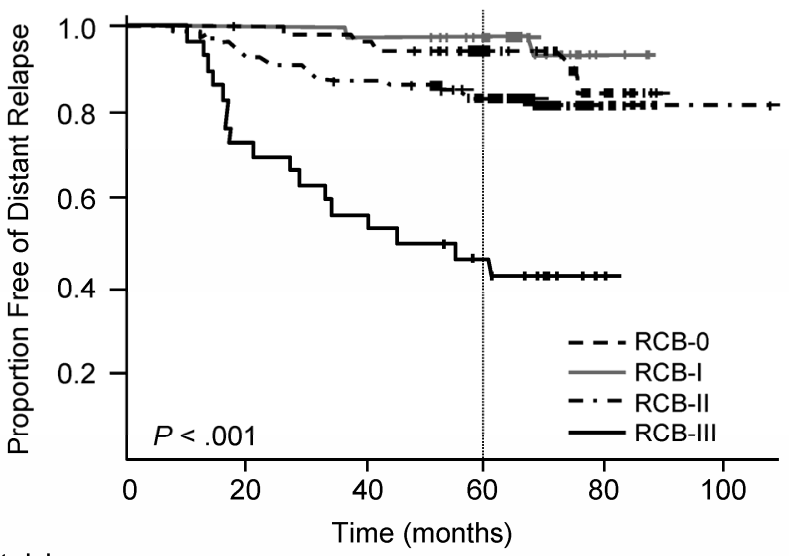

No. at risk

$\begin{array}{lrrrrrr}\text { RCB-0 } & 55 & 55 & 54 & 43 & 14 & 1 \\ \text { RCB-I } & 42 & 42 & 41 & 35 & 4 & 1 \\ \text { RCB-II } & 114 & 106 & 99 & 78 & 13 & 2 \\ \text { RCB-III } & 30 & 23 & 18 & 13 & 2 & 1\end{array}$

Figure 1: Likelihood of distant relapse in patients with residual cancer burden (RCB) -0 (pCR), RCB-I (npCR, minimal residual disease), RCB-II (moderate residual disease), or RCB-III (extensive residual disease). $P$ value is from a log-rank test for difference between all survival curves. RCB is calculated as a continuous index combining pathologic measurements of primary tumor (size and cellularity) and nodal metastases (number and size) to predict distant relapse-free survival (calculator available online at: http://www.mdanderson.org/breastcancer RCB). Reproduced with permission from Symmans et al. 2007 [13].

\section{Capecitabine plus docetaxel for early $B C$}

Adding a taxane to anthracycline-based preoperative chemotherapy significantly improves overall response rates (ORR) and pCR rates $[8,16]$. Combinations of capecitabine and a taxane are highly active in metastatic breast cancer (MBC) [17] and provide a promising non-anthracycline-based alternative for patients with early breast cancer with the advantage of potentially less cardiotoxicity [18].

Capecitabine is an oral fluoropyrimidine designed to deliver 5-fluorouracil (5-FU) selectively to tumor sites by exploiting higher concentrations of thymidine phosphorylase (TP) in malignant cells compared with normal cells [19]. TP activity is upregulated by several chemotherapeutic agents, including taxanes, providing an elegant explanation for the preclinical synergy between capecitabine and docetaxel [20-22]. TP expression may also be a predictive marker for the clinical benefit of docetaxel plus capecitabine in patients with breast cancer [23].

In a randomized trial in patients with $\mathrm{MBC}$, the combination of capecitabine $1250 \mathrm{mg} / \mathrm{m}^{2}$ twice daily (BID) on days $1-14$ plus docetaxel $75 \mathrm{mg} / \mathrm{m}^{2}$ on day 1 (XT) significantly improved OS (hazard ratio, 0.775; 95\% confidence interval [CI], 0.634-0.947) compared with docetaxel alone [17]. Gastrointestinal side effects and hand-foot syndrome were more common with XT, whereas myalgia, arthralgia, and neutropenic fever/sepsis were more common with single-agent docetaxel. Based on clinical experience and findings from retrospective analyses of dose modification in this trial, lower doses of capecitabine and docetaxel are typically used in this setting and do not appear to reduce efficacy $[24,25]$. The high activity and manageable safety profile of XT given at appropriate doses in the metastatic setting provided the rationale for evaluating $\mathrm{XT}$ in early breast cancer [26].

In a phase III trial, 209 women with axillary node-positive, stage II/III breast cancer were randomly allocated to receive four 3-weekly cycles of preoperative XT (capecitabine $1000 \mathrm{mg} / \mathrm{m}^{2}$ BID on days $1-14$ and docetaxel $75 \mathrm{mg} / \mathrm{m}^{2}$ on day 1 ) or $\mathrm{AC}$ (doxorubicin $60 \mathrm{mg} / \mathrm{m}^{2}$ and cyclophosphamide 600 $\mathrm{mg} / \mathrm{m}^{2}$ on day 1) [18]. XT significantly increased $\mathrm{pCR}$ rate $(21 \%$ vs. $10 \%$, respectively, $P=0.024)$ and ORR ( $84 \%$ vs. $65 \%$, respectively, $P=0.003$ ). Of note, the pCR rate achieved with $\mathrm{XT}$ was within the range seen with anthracycline-taxane sequential therapy in a mixed population of patients (human epidermal growth factor receptor [HER]2 negative and positive) [8]. Since most of the published preoperative trials used 6 or 8 cycles of treatment, it was considered important to explore the activity of a shorter treatment course (4 cycles) for both HER2-positive and HER2-negative patients. 
Capecitabine, docetaxel, plus trastuzumab for HER2-positive BC

Non-anthracycline preoperative regimens are a particularly interesting proposition in HER2-positive breast cancer, because they avoid the risk of anthracycline-induced cardiotoxicity in patients eligible for adjuvant or preoperative trastuzumab. The value of preoperative trastuzumab plus chemotherapy has been demonstrated in several phase II studies [9,27-31]. In 42 patients with operable breast cancer, adding trastuzumab to preoperative paclitaxel followed by 5-FU, epirubicin, and cyclophosphamide significantly improved $\mathrm{pCR}$ rate compared with chemotherapy alone $(67 \%$ vs. $25 \%$, respectively; $P=0.02)$ [30].

In vivo data [32] and clinical studies [33-35] have demonstrated the efficacy of trastuzumab plus capecitabine in HER2-positive breast cancer. A randomized phase II study in patients with MBC or LABC compared 3-weekly cycles of HXT (trastuzumab $8 \mathrm{mg} / \mathrm{kg}$ loading dose followed by $6 \mathrm{mg} / \mathrm{kg}$, capecitabine 950 $\mathrm{mg} / \mathrm{m}^{2}$ BID days $1-14$, and docetaxel $75 \mathrm{mg} / \mathrm{m}^{2}$ ) with HT (trastuzumab and docetaxel $100 \mathrm{mg} / \mathrm{m}^{2}$ ) [36]. Both combinations produced high ORR $(71 \%$ and $73 \%$, respectively), but the HXT combination significantly prolonged both time to progression and progression-free survival compared with HT, with the median increased by 5 months for both parameters. Promising results were also observed in a phase II study of preoperative HXT (trastuzumab, capecitabine $900 \mathrm{mg} / \mathrm{m}^{2}$ BID, and docetaxel $36 \mathrm{mg} / \mathrm{m}^{2}$ days 1 and 8) administered every 3 weeks to patients with HER2-positive LABC (or XT alone for patients with HER2-negative tumors) [37]. The ORR was $94 \%$ and, in patients treated with HXT, the pCR rate was $45 \%$. HXT demonstrated good tolerability in these studies: the lower $\mathrm{XT}$ dose is well tolerated, and HXT may reduce the risk of overlapping cardiac toxicity with adjuvant anthracyclines.

\section{XeNA}

This open-label, multicenter, phase II study uses Simon's optimal two-stage design [38] to independently assess the efficacy of preoperative XT in HER2-negative and HXT in HER2-positive breast cancer. The study design was approved by the ethics committees at participating institutions, and all patients provided written informed consent. Enrollment of 157 patients was completed in May 2007; 156 patients (122 HER2-negative and 34 HER2-positive) are evaluable.

\section{Patient Population}

Women $\geq 18$ years with newly diagnosed, his- tologically confirmed, infiltrating (invasive), HER2-negative or HER2-positive stage II/III breast cancer, with no evidence of metastatic disease except ipsilateral axillary lymph nodes (T2-3, N0-1, M0), were eligible for the study provided that they had not previously received any type of systemic or local primary treatment for breast cancer. To facilitate response assessment, patients were required to have a clinically palpable tumor of $>2 \mathrm{~cm}$, meeting the Response Evaluation Criteria for Solid Tumors (RECIST) for palpable measurable disease. Key exclusion criteria included inflammatory breast cancer, clinically significant cardiac disease, and inadequate renal function.

\section{Treatments}

All eligible patients received four 21-day cycles of capecitabine $825 \mathrm{mg} / \mathrm{m}^{2}$ BID on days 1-14 plus docetaxel $75 \mathrm{mg} / \mathrm{m}^{2}$ on day 1 . This dosing regimen was selected on the basis of previous clinical studies of XT suggesting that doses can be reduced to improve tolerability without adversely affecting efficacy [24,26]. Patients with HER2-positive tumors determined by fluorescence in situ hybridization (FISH) also received a trastuzumab $4 \mathrm{mg} / \mathrm{kg}$ loading dose (90-minute infusion) on day 1 followed by $2 \mathrm{mg} / \mathrm{kg}$ weekly (30-minute infusion) for 11 doses (Figure 2). An Independent Data Monitoring Board evaluated the safety and initial efficacy after treatment of a predetermined number of patients. Based on their analysis of the data, the trial was allowed to continue with no change to treatment doses and schedules; a change in the efficacy endpoint to $\mathrm{PCR}$ plus npCR was suggested.

\section{Primary and Secondary End Points}

The primary endpoint for the study was the rate of pCR plus npCR (residual T1a) in the affected breast after 4 cycles of preoperative therapy, determined by pathologic assessment of the resected specimen at the time of definitive surgery. A hematoxylin and eosin stained slide from each of the paraffin blocks prepared from the breast tissue and lymph nodes was reviewed at a central laboratory (Albany Medical College Department of Pathology and Laboratory Medicine, New York, NY) to establish the presence or absence of infiltrating breast cancer. Absence of histological evidence of invasive breast cancer cells defined pCR; npCR was defined as the presence of invasive tumor $\leq 5 \mathrm{~mm}$ (T1a). While pCR and npCR appear to have equivalent power to predict long-term survival [13], the sum of these endpoints has the advantage of providing a greater number of informative events, which is a particularly important consideration for the complementary correlative studies described below.

Secondary clinical endpoints defined for the study included pCR and $n p C R$ as individual efficacy 
parameters, ORR (according to RECIST), rates of local recurrence, DFS, OS, safety profile (assessed using National Cancer Institute Common Terminology Criteria for Adverse Events, version 3.0), and quality of life (measured by the Functional Assessment of Cancer Therapy - Breast before, during, and at the completion of preoperative therapy).

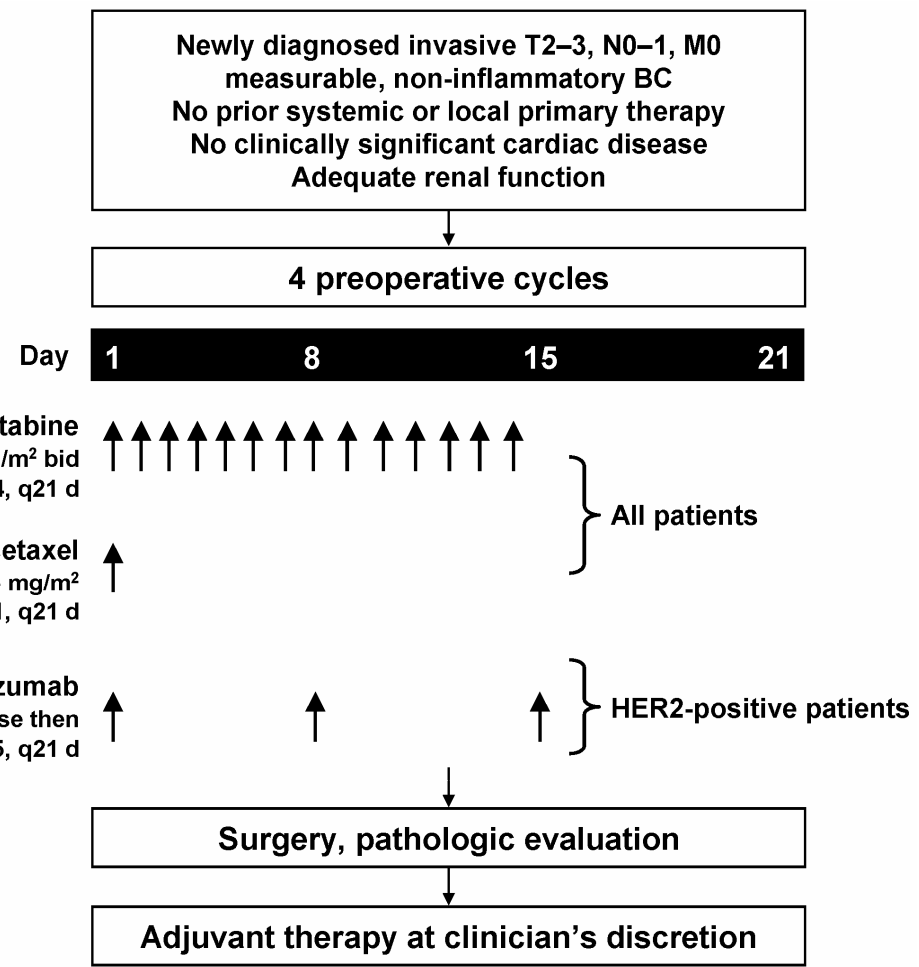

Figure 2: Treatment schedule.

\section{Correlative Studies}

Blood and tissue biomarker levels and candidate gene expression were measured prior to preoperative therapy and at the time of surgery to identify predictive factors associated with pathologic response to XT or HXT. Potential biomarkers include TP, thymidine synthase (TS), and dihydropyrimidine dehydrogenase (DPD) for capecitabine and Tau protein for taxanes. Circulating tumor cell (CTC) levels were determined using the CellSearch System (Veridex, LLC., Warren, NJ) prior to the first treatment cycle and prior to definitive surgery in patients with HER2 positive tumors. An interim analysis confirmed that CTCs were detectable in patients in this study. Microarray analysis (AmpliChip p53 test; Roche Diagnostics, Indianapolis, IN) was used to measure p53 gene mutation levels in tissue samples prior to treatment. Preliminary data showed that approximately half of patients expressed p53 mutations at diagnosis.

\section{Interim Analysis}

Baseline characteristics of 90 patients included in an interim analysis are shown in Table 1 [39]. The clinical response rates were 79\% in HER2-negative and 90\% in HER2-positive breast cancer. The rate of pCR plus npCR was 52\% in patients with HER2-positive BC. After four treatment cycles, mean tumor diameters were reduced by $55 \%$ and $61 \%$, respectively, in the HER2-negative and HER2-positive groups. The most frequent adverse events were hematologic toxicities and hand-foot syndrome. There were no grade 4 adverse events, treatment-related deaths, or clinical or subclinical cardiac events. Treatment was discontinued because of toxicity in 15 patients $(10 \%)$ and four patients progressed prior to surgery.

Table 1. Characteristics of patients included in an interim analysis.

\begin{tabular}{lll}
\hline Baseline characteristics & $\begin{array}{l}\text { HER2-negative } \\
(\mathrm{N}=67)\end{array}$ & $\begin{array}{l}\text { HER2-positive } \\
(\mathrm{N}=23)\end{array}$ \\
Median age, years (range) & $52(29-82)$ & $52(32-66)$ \\
\hline Estrogen receptor positive & $44(65 \%)$ & $10(43 \%)$ \\
Mean tumor diameter, cm & 5.5 & 5.6 \\
\hline
\end{tabular}




\section{CONCLUSIONS}

XT and HXT are promising non-anthracycline regimens for the preoperative treatment of women with HER2-negative and HER2-positive breast cancer, respectively. The ongoing XeNA study, in which treatment was assigned based on centrally performed FISH analysis, is expected to provide further clinical evidence to support the high activity observed with these regimens in patients with LABC or MBC $[17,36]$. A particularly important feature of the XeNA study is the use of $\mathrm{pCR}$ plus $\mathrm{npCR}$ as the primary endpoint. This endpoint is justified by previous data showing that patients with T1a tumors have excellent long-term survival [12] and that $\mathrm{pCR}$ and $\mathrm{npCR}$ have equivalent predictive power [13]. In addition, by increasing the number of informative events, the sum of $\mathrm{pCR}$ and npCR will facilitate correlative studies designed to identify patients most likely to benefit from $\mathrm{XT}$ and HXT and may expedite the clinical evaluation of these novel preoperative regimens. The final analysis, including data from these correlative studies specifically designed to identify predictive biomarkers associated with pathologic response, will be available in 2009.

\section{ACKNOWLEDGEMENTS}

The XeNA study is sponsored by Roche, Nutley, NJ, USA. Medical writing support was provided by Tim Kelly for Insight Medical Communications, Inc., a division of Grey Healthcare Group, on behalf of Roche, Nutley, NJ, USA.

\section{CONFLICT OF INTEREST}

Dr. Gluck received honoraria, consultant and research funding for this study from Roche, Genentech, and Sanofi-Aventis.

Edward F. McKenna, Jr, PharmD, is an employee of Roche (Associate Medical Director, Oncology).

Dr. Royce received grant support from Roche and Genentech and is a member of the speaker's bureau for Genentech and Roche.

\section{REFERENCES}

1. Kaufmann M, Hortobagyi GN, Goldhirsch A, et al. Recommendations from an international expert panel on the use of neoadjuvant (primary) systemic treatment of operable breast cancer: an update. J Clin Oncol. 2006; 24:1940-1949.

2. Fisher B, Bryant J, Wolmark N, et al. Effect of preoperative chemotherapy on the outcome of women with operable breast cancer. J Clin Oncol. 1998; 16:2672-2685.

3. Mauri D, Pavlidis N, Ioannidis JP. Neoadjuvant versus adjuvant systemic treatment in breast cancer: a meta-analysis. J Natl Cancer Inst. 2005; 97:188-94.

4. Sachelarie I, Grossbard ML, Chadha M, et al. Primary systemic therapy of breast cancer. Oncologist. 2006; 11:574-589.

5. Bonadonna G, Valagussa P, Brambilla C, et al. Primary chemotherapy in operable breast cancer: eight-year experience at the Milan Cancer Institute. J Clin Oncol. 1998; 16:93-100.
6. Kuerer HM, Newman LA, Smith TL, et al. Clinical course of breast cancer patients with complete pathologic primary tumor and axillary lymph node response to doxorubicin-based neoadjuvant chemotherapy. J Clin Oncol. 1999; 17:460-469.

7. Ogston KN, Miller ID, Payne S, et al. A new histological grading system to assess response of breast cancers to primary chemotherapy: prognostic significance and survival. Breast. 2003; 12:320-327.

8. Bear HD, Anderson S, Smith RE, et al. Sequential preoperative or postoperative docetaxel added to preoperative doxorubicin plus cyclophosphamide for operable breast cancer: National Surgical Adjuvant Breast and Bowel Project Protocol B-27. J Clin Oncol. 2006; 24:2019-2027.

9. Hurley J, Doliny P, Reis I, et al. Docetaxel, cisplatin, and trastuzumab as primary systemic therapy for human epidermal growth factor receptor 2-positive locally advanced breast cancer. J Clin Oncol. 2006; 24:1831-1838.

10. Kuroi $\mathrm{K}$, Toi M, Tsuda $\mathrm{H}$, et al. Issues in the assessment of the pathologic effect of primary systemic therapy for breast cancer. Breast Cancer. 2006; 13:38-48.

11. Mazouni C, Peintinger F, Wan-Kau S, et al. Residual ductal carcinoma in situ in patients with complete eradication of invasive breast cancer after neoadjuvant chemotherapy does not adversely affect patient outcome. J Clin Oncol. 2007; 25:2650-2655.

12. Rosen PP, Groshen S, Kinne DW, Norton L. Factors influencing prognosis in node-negative breast carcinoma: analysis of 767 T1N0M0/T2N0M0 patients with long-term follow-up. J Clin Oncol. 1993; 11:2090-2100.

13. Symmans WF, Peintinger F, Hatzis C, et al. Measurement of residual breast cancer burden to predict survival after neoadjuvant chemotherapy. J Clin Oncol. 2007; 25:4414-4422.

14. Peintinger F, Buzdar AU, Kuerer HM, et al. Hormone receptor status and pathologic response of HER2-positive breast cancer treated with neoadjuvant chemotherapy and trastuzumab. Ann Oncol. 2008; [Epub ahead of print]

15. Carey LA, Metzger R, Dees EC, et al. American Joint Committee on Cancer tumor-node-metastasis stage after neoadjuvant chemotherapy and breast cancer outcome. J Natl Cancer Inst. 2005; 97:1137-1142.

16. Heys SD, Hutcheon AW, Sarkar TK, et al. Neoadjuvant docetaxel in breast cancer: 3-year survival results from the Aberdeen trial. Clin Breast Cancer. 2002; 3(Suppl 2):S69-74.

17. O'Shaughnessy J, Miles D, Vukelja S, et al. Superior survival with capecitabine plus docetaxel combination therapy in anthracycline-pretreated patients with advanced breast cancer: phase III trial results. J Clin Oncol. 2002; 20:2812-2823.

18. Lee KS, Ro J, Nam BH, et al. A randomized phase-III trial of docetaxel/capecitabine versus doxorubicin/cyclophosphamide as primary chemotherapy for patients with stage II/III breast cancer. Breast Cancer Res Treat. 2008; 109:481-489.

19. Miwa M, Ura M, Nishida M, et al. Design of a novel oral fluoropyrimidine carbamate, capecitabine, which generates 5-fluorouracil selectively in tumours by enzymes concentrated in human liver and cancer tissue. Eur J Cancer. 1998; 34:1274-1281.

20. Sawada N, Ishikawa T, Fukase Y, et al. Induction of thymidine phosphorylase activity and enhancement of capecitabine efficacy by taxol/taxotere in human cancer xenografts. Clin Cancer Res. 1998; 4:1013-1019.

21. Toi M, Bando H, Horiguchi S, et al. Modulation of thymidine phosphorylase by neoadjuvant chemotherapy in primary breast cancer. Br J Cancer. 2004; 90:2338-2343.

22. Puglisi F, Andreetta C, Valent F, et al. Anthracyclines and taxanes induce the upregulation of thymidine phosphorylase in breast cancer cells. Anticancer Drugs. 2007; 18: 883-888.

23. Puglisi F, Cardellino GG, Crivellari D, et al. Thymidine phosphorylase expression is associated with time to progression in 
patients receiving low-dose, docetaxel-modulated capecitabine for metastatic breast cancer. Ann Oncol. 2008;19:1541-1546.

24. Leonard R, O'Shaughnessy J, Vukelja S, et al. Detailed analysis of a randomized phase III trial: can the tolerability of capecitabine plus docetaxel be improved without compromising its survival advantage? Ann Oncol. 2006; 17:1379-1385.

25. Silva O, Lopes G, Morgenzstern D, et al. A phase II trial of split, low dose docetaxel and low dose capecitabine: a tolerable and efficacious regimen in the first line treatment of patients with Her2Neu-negative metastatic breast cancer. Clin Breast Cancer. 2008; 8:162-167.

26. Lebowitz PF, Eng-Wong J, Swain SM, et al. A phase II trial of neoadjuvant docetaxel and capecitabine for locally advanced breast cancer. Clin Cancer Res. 2004; 10:6764-6769.

27. Coudert BP, Largillier R, Arnould L, et al. Multicenter phase II trial of neoadjuvant therapy with trastuzumab, docetaxel, and carboplatin for human epidermal growth factor receptor-2-overexpressing stage II or III breast cancer: results of the GETN(A)-1 trial. J Clin Oncol. 2007; 25:2678-2684.

28. Wenzel C, Hussian D, Bartsch R, et al. Preoperative therapy with epidoxorubicin and docetaxel plus trastuzumab in patients with primary breast cancer: a pilot study. J Cancer Res Clin Oncol. 2004; 130:400-404.

29. Burstein HJ, Harris LN, Gelman R, et al. Preoperative therapy with trastuzumab and paclitaxel followed by sequential adjuvant doxorubicin/cyclophosphamide for HER2 overexpressing stage II or III breast cancer: a pilot study. J Clin Oncol. 2003; 21:46-53.

30. Buzdar AU, Ibrahim NK, Francis D, et al. Significantly higher pathologic complete remission rate after neoadjuvant therapy with trastuzumab, paclitaxel, and epirubicin chemotherapy: results of a randomized trial in human epidermal growth factor receptor 2-positive operable breast cancer. J Clin Oncol. 2005; 23:3676-3685.

31. Buzdar AU, Valero V, Ibrahim NK, et al. Neoadjuvant therapy with paclitaxel followed by 5-fluorouracil, epirubicin, and cyclophosphamide chemotherapy and concurrent trastuzumab in human epidermal growth factor receptor 2-positive operable breast cancer: an update of the initial randomized study population and data of additional patients treated with the same regimen. Clin Cancer Res. 2007; 13:228-233.

32. Fujimoto-Ouchi K, Sekiguchi F, Tanaka Y. Antitumor activity of combinations of anti-HER-2 antibody trastuzumab and oral fluoropyrimidines capecitabine/5'-dFUrd in human breast cancer models. Cancer Chemother Pharmacol. 2002; 49:211-216.

33. Schaller G, Fuchs I, Gonsch T, et al. Phase II study of capecitabine plus trastuzumab in human epidermal growth factor receptor 2 overexpressing metastatic breast cancer pretreated with anthracyclines or taxanes. J Clin Oncol. 2007; 25:3246-3250.

34. Yamamoto D, Iwase S, Kitamura K, Odagiri H, Yamamoto C, Nagumo Y. A phase II study of trastuzumab and capecitabine for patients with HER2-overexpressing metastatic breast cancer: Japan Breast Cancer Research Network (JBCRN) 00 Trial. Cancer Chemother Pharmacol. 2007; 61:509-514.

35. Bartsch R, Wenzel C, Altorjai G, et al. Capecitabine and trastuzumab in heavily pretreated patients with metastatic breast cancer. J Clin Oncol. 2007; 25:3853-3858.

36. Wardley A, Antón-Torres A, Pivot X, et al. Evaluation of trastuzumab, docetaxel and capecitabine as first-line therapy for HER2-positive locally advanced or metastatic breast cancer. Breast Cancer Res Treat. 2007; 106(Suppl 1):S33.

37. Lybaert W, Wildiers H, Neven P, et al. Multicenter phase II study of neoadjuvant capecitabine $(\mathrm{X})$, docetaxel $(\mathrm{T}) \pm \operatorname{trastuzumab}(\mathrm{H})$ for patients (pts) with locally advanced breast cancer (LABC): preliminary safety and efficacy data. Breast Cancer Res Treat. 2006; 100(Suppl 1):S147-S148.
38. Simon R. Optimal two-stage designs for phase II clinical trials. Controlled Clin Trials. 1989; 10:1-10.

39. Tripathy D, Moisa C, Gluck S. Neoadjuvant capecitabine plus docetaxel \pm trastuzumab therapy for recently diagnosed breast cancer: phase II results. Breast Cancer Res Treat. 2007; 106(Suppl 1):S226. 